\title{
Respiratory pattern formation in the isolated bullfrog (Rana catesbeiana) brainstem-spinal cord
}

\author{
Stephen G. Reid *, William K. Milsom \\ Department of Zoology, University of British Columbia, 6270 University Boulevard, Vancouver, B.C. V6T 1Z4, Canada
}

Accepted 14 September 1998

\begin{abstract}
This study characterizes various patterns of motor output obtained from cranial nerves V, VII, X, and XII of in vitro, saline-perfused, brainstem-spinal cord preparations of the American bullfrog (Rana catesbeiana). Motor output indicative of fictive breathing was present in all preparations. In 17 of 26 preparations, fictive breaths were either evenly spaced or randomly distributed, while in the remaining nine preparations fictive breaths occurred in episodes separated by relatively long periods of quiescence. With the exception of fictive breath duration in the non-episodic preparations and the instantaneous frequency of fictive breaths within episodes, all variables associated with fictive breathing were insensitive to changes in perfusion saline $\mathrm{pH}$. In addition to fictive breathing, a large number of other forms of motor output were observed arising from these nerves. While the data suggest that the in vitro preparation is capable of producing a wide repertoire of motor patterns, similar to those seen in vivo, it was difficult, with the current protocol, to reliably produce any single pattern in spite of carefully regulated conditions. C) 1998 Elsevier Science B.V. All rights reserved.
\end{abstract}

Keywords: Amphibians, bullfrog (Rana catesbeiana); Brainstem, central pattern generation; Control of breathing; Pattern of breathing, generation, episodic breathing

* Corresponding author: Present address: Department of Biology, McMaster University, 1280 Main Street West, Hamilton, Ontario, L8S 4K1 Canada. Tel.: + 1-905-5259140 ext. 27389/27412; fax: +1-905-5226066; e-mail: reidsg@ mcmail.mcmaster.ca.

\section{Introduction}

In vitro, brainstem-spinal cord preparations are currently being used to study the control of breathing in numerous vertebrate species, particularly mammals (e.g. Suzue, 1984; Feldman et al., 1991). One of the primary applications of such preparations is the elucidation of mechanisms responsible for the genesis, at the cellular level, of 
respiratory rhythm (e.g. Feldman and Smith, 1989). Recently, such investigations have examined central mechanisms underlying the control of breathing in lower vertebrates including both adult and larval amphibians (Kinkead et al., 1994; McLean et al., 1995a,b; Galante et al., 1996; Liao et al., 1996; Torgerson et al., 1997a,b). These studies show that spontaneous rhythmical bursting activity, representative of the efferent motor output underlying lung ventilation, can be recorded from trigeminal, facial, vagal, and hypoglossal nerve roots in the isolated brainstem of both tadpoles and adult frogs. They conclude that burst frequency, burst duration, and the activity profile of the spontaneous neural discharge resembles that recorded from decerebrate, fictively breathing animals (Sakakibara, 1984a,b; Kinkead and Milsom, 1994; Kogo and Remmers, 1994; Kogo et al., 1994; Perry et al., 1995; Kinkead et al., 1997; Kinkead and Milsom, 1997).

Another potential application of this preparation is the study of the mechanisms responsible for the production of breathing pattern. The pattern of motor output to the respiratory muscles arises from the interaction of central rhythm generator activity with various sensory inputs (chemoreceptors, lung mechanoreceptors, etc.) and descending central influences (hypothalmus, reticular formation, etc.) (Feldman et al., 1990). The central $\mathrm{CO}_{2} / \mathrm{H}^{+}$chemoreceptors are still present in this preparation (Kinkead et al., 1994; McLean et al., 1995a,b) as is the nucleus isthmi, an area of the midbrain suggested to play a role in breathing pattern formation (Kinkead et al., 1997). Furthermore, it has been shown that stimulation of the cut vagus nerve roots can, to some extent, mimic the effect of pulmonary receptor input (Kinkead et al., 1994). Given this, however, it is interesting to note that studies performed to date on adult frogs (Kinkead et al., 1994; McLean et al., 1995a,b) have produced very different breathing patterns and, in all cases, the $\mathrm{CO}_{2} / \mathrm{H}^{+}$ sensitivity of the preparations has been exceedingly blunted compared with that of vagally stimulated preparations, in situ preparations or intact adult animals. This is surprising but both large variability and insensitivity were also hallmarks of this preparation in preliminary studies in our hands (Reid and Milsom, 1996). Thus, in the present study, we have endeavored to quantify the nature and extent of breathing pattern variation found in the in vitro brainstem-spinal cord preparation of the adult American bullfrog in a continuing effort to evaluate the utility of this preparation as a tool to study the control of breathing pattern formation in amphibians.

\section{Materials and methods}

\subsection{Experimental animals}

Bullfrogs (Rana catesbeiana) (250g; $n=26$ ) were obtained from a commercial supplier (Cyr's Biological Company, Ponchatoula, LA) and transported to the University of British Columbia. Animals were maintained in fiberglass tanks supplied with a constant flow of dechlorinated City of Vancouver tapwater to provide a moist environment. Room temperature was approximately $20^{\circ} \mathrm{C}$ and the photoperiod was maintained at 12L:12D. Frogs were fed live locusts at least once a week. The animals were allowed to acclimate to laboratory conditions for a minimum of one week prior to experimentation.

\subsection{The in vitro brainstem-spinal cord preparation}

Bullfrogs were anaesthetized by partial submersion in an aqueous solution of ethyl- $m$-aminobenzoate (MS 222; $0.6 \mathrm{~g} / 1$ ) buffered to $\mathrm{pH} 7.0$ with sodium bicarbonate. A surgical level of anesthesia (i.e. elimination of withdrawal and eye blink reflexes) was obtained in $\approx 30 \mathrm{~min}$. A longitudinal incision was made in the skin above the cranium and the skin removed. Using a dental drill, a small hole was made in the skull rostral to the optic lobes and the cranial case was removed with bone shears and placed onto a Sylgard-coated dissecting dish. The brain was exposed, perfusion with oxygenated saline (see below) was initiated, and the rostral forebrain was removed. The remaining tissue was continually supplied with a constant perfusion of oxygenated, bicarbonatebuffered saline (in mmol/1; $\mathrm{NaCl}, 75.0 ; \mathrm{KCl}, 4.5$; 
$\mathrm{MgCl}_{2}, 1.0 ; \mathrm{NaH}_{2} \mathrm{PO}_{4}, 1.0 ; \mathrm{NaHCO}_{3}, 40.0 ; \mathrm{CaCl}_{2}$, 2.5; glucose, 5.0; pH 7.8; Kinkead et al., 1994). A $\mathrm{pH}$ of 7.8 was selected as it approximates the $\mathrm{pH}$ of amphibian plasma at room temperature (West et al., 1987; McLean et al., 1995a).

Cranial nerves were cut close to their exit from the skull and the spinal cord was severed at the level of the second spinal nerve. The brainstemspinal cord was lifted from the cranial case and pinned in a dissecting dish where the membranes (dural, arachnoid, meninges) were removed in order to free the cranial nerve roots. The tips of the nerves were cut in order to provide a clean, undamaged surface for recording. The preparation was then pinned, ventral side up, on a fine stainless steel mesh within a perfusion chamber. The mesh divided the chamber into upper and lower compartments, which facilitated constant perfusion of both surfaces (Kinkead et al., 1994; McLean et al., 1995a,b). Once the brainstemspinal cord was secure within the chamber $(\approx 30$ min following the commencement of surgery), the preparation was perfused, utilizing a flow through system, ( $\mathrm{pH} 7.8 ; 5 \mathrm{ml} / \mathrm{min})$ for at least $1 \mathrm{~h}$ to allow it to 'stabilize'. All experiments were performed at room temperature. The small diffusion distances within the brainstem, coupled with its anoxia-hypoxia tolerance, allow this preparation to remain viable for many hours. Indeed, this preparation may remain viable for approximately one week if maintained overnight at $0^{\circ} \mathrm{C}$ in an oxygen enriched atmosphere (McLean et al., 1995a,b; Perry et al., 1995).

\subsection{Fictive breathing and experimental protocol}

Utilizing a micro-manipulator, a suction electrode, synthesized from thin-walled capillary glass (inner diameter $1 \mathrm{~mm}$ ) was positioned near the end of one severed nerve and the nerve rootlet was gently aspired into the electrode. Numerous electrodes, with a wide range of tip diameters, were fashioned in order to provide an assortment of electrodes such that one of appropriate size was always available to obtain a tight seal on different nerve roots from animals of different size.

Care was taken to ensure that the nerve fit tightly into the electrode permitting the formation of a good seal. Once the nerve rootlet was firmly in place within the electrode, whole nerve discharge (motor output) was recorded as an index of fictive breathing. The majority of the recordings were obtained from the trigeminal (22 preparations) or vagus (20) nerves although the facial (5), and hypoglossal (4) nerves were used in some instances.

Gassing with varying levels of $\mathrm{CO}_{2}$ altered the $\mathrm{pH}$ of the perfusion saline to levels between 7.1 and 8.0. The $\mathrm{pH}$ of the mock CSF was measured with a $\mathrm{pH}$ meter/electrode (Corning) with the electrode positioned in the perfusate reservoir. These values approximated the $\mathrm{pH}$ range utilized by McLean et al. (1995a; 7.4-8.6). However, given the reduced response to a decrease in $\mathrm{pH}$ in the present study, the acidic range was extended and the alkaline range truncated.

Nerve discharge was observed in these preparations within 1-6 h post-dissection. This delay presumably reflects the time required for residual anaesthetic to diffuse from the tissue. Once stable levels of neural discharge were observed, motor output was then recorded for a period of 10-30 min (depending upon the constancy of the pattern(s) of motor output) at $\mathrm{pH}$ 7.8. The $\mathrm{pH}$ was then lowered by $0.1 \mathrm{pH}$ unit (lower limit of $\mathrm{pH}$ $7.1-7.2)$ followed by the equilibration $(30 \mathrm{~min})$ and recording $(10-30 \mathrm{~min})$ periods. In some preparations the initial $\mathrm{pH}$ step was from 7.8 to 8.0 followed by the stepwise decrease to $\mathrm{pH} 7.1-$ 7.2 .

\subsection{Data acquisition and analysis}

Nerve activity from the suction electrode was amplified (filter settings: $50 \mathrm{~Hz}$ (high pass) and 10 $\mathrm{kHz}$ (low pass)), full wave rectified and integrated. The signal was monitored visually using an oscilloscope (Tektronix 5111A) and acoustically with an audio monitor (Grass AM8). Activity was recorded on a chart recorder and representative recordings were saved using a data acquisition system (Windaq, DataQ Systems). The sampling rate of analogue to digital conversion was 2000 $\mathrm{Hz}$.

Breathing episodes were designated according to the criteria of Kinkead and Milsom (1997). The 


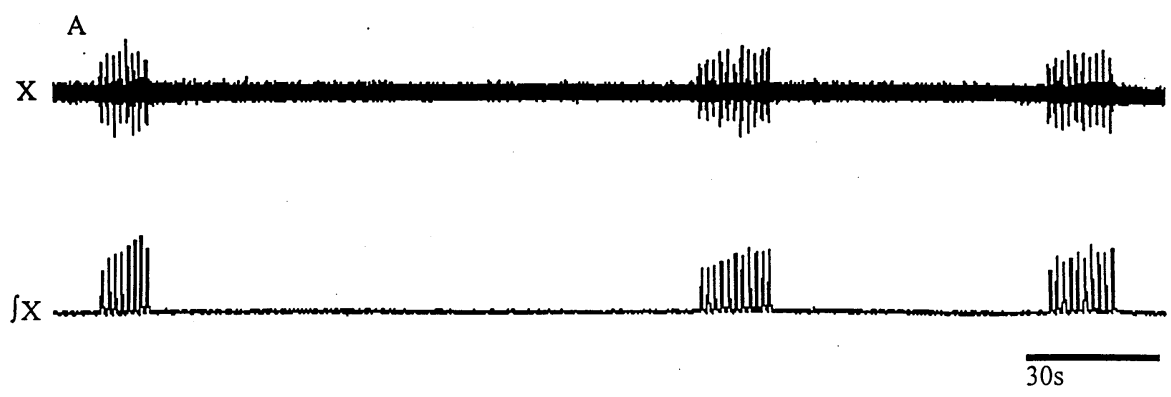

B
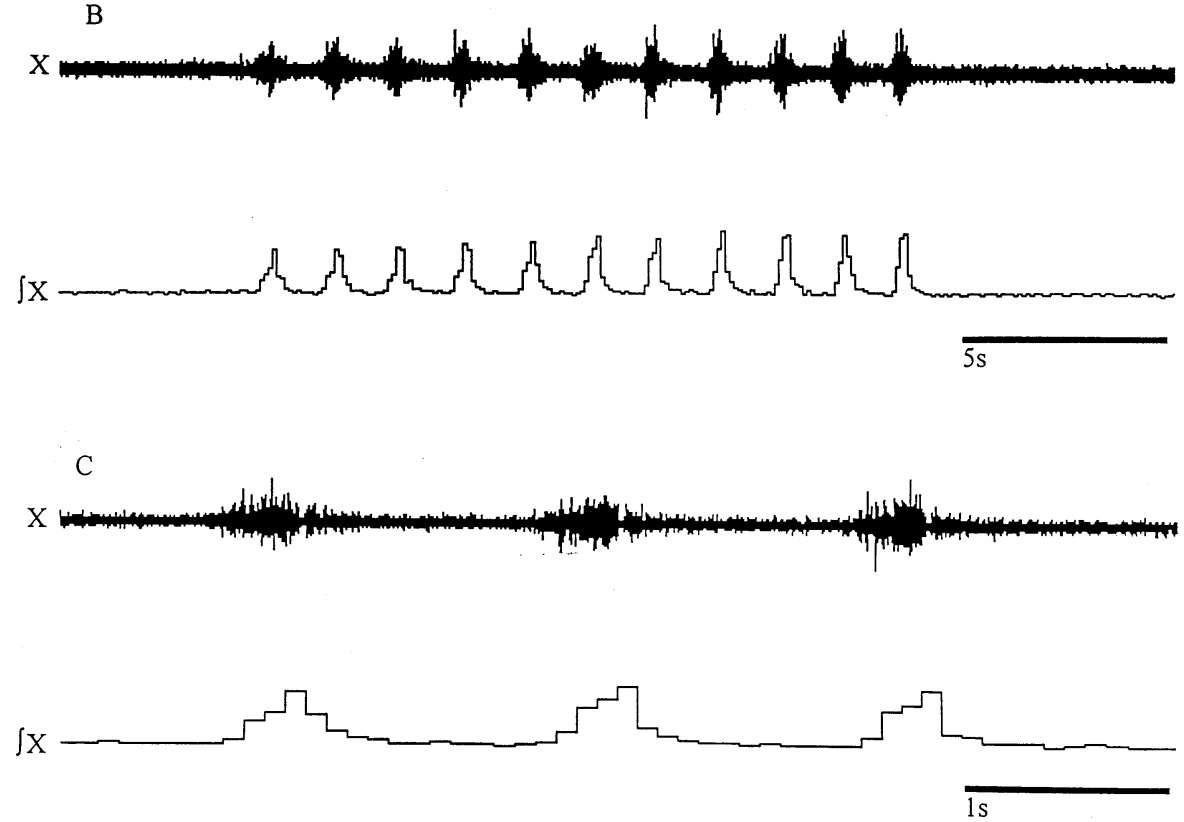

Fig. 1. Episodic motor output recorded from the vagus $(\mathrm{X})$ nerve root in the isolated bullfrog brainstem-spinal cord preparation. In each trace the upper figure illustrates the full wave rectified signal while the lower trace illustrates the integrated ( $\int \mathrm{X}$ ) signal. (A) Three individual episodes of fictive breaths from the vagus nerve. (B) An expanded trace of one of the individual episodes of fictive breaths illustrated in A. (C) A further expanded trace illustrating three individual fictive breaths from the previous panel.

number of breaths within an episode was obtained by counting the number of fictive breaths that occurred in succession with no pause longer than the length of two ventilation cycles between them. When breathing was continuous, no values were obtained for breaths per episode or episodes per minute. Breathing frequency was quantified by analyzing the number of fictive breaths per unit time (absolute frequency; breaths/min) as well as by measuring the period between two successive, uninterrupted breaths within an episode. The inverse of this period was then multiplied by 60 to obtain the instantaneous frequency (breaths $/ \mathrm{min}$ ).

The classification of neural discharge as a fictive breath or 'other' discharge is based on comparisons of the in vitro motor output to fictive breathing obtained in a less reduced preparation, the decerebrate, paralyzed and unidirectionally-ventilated in situ preparation. As such, in this study, respiratory related discharge (i.e. a fictive breath) 

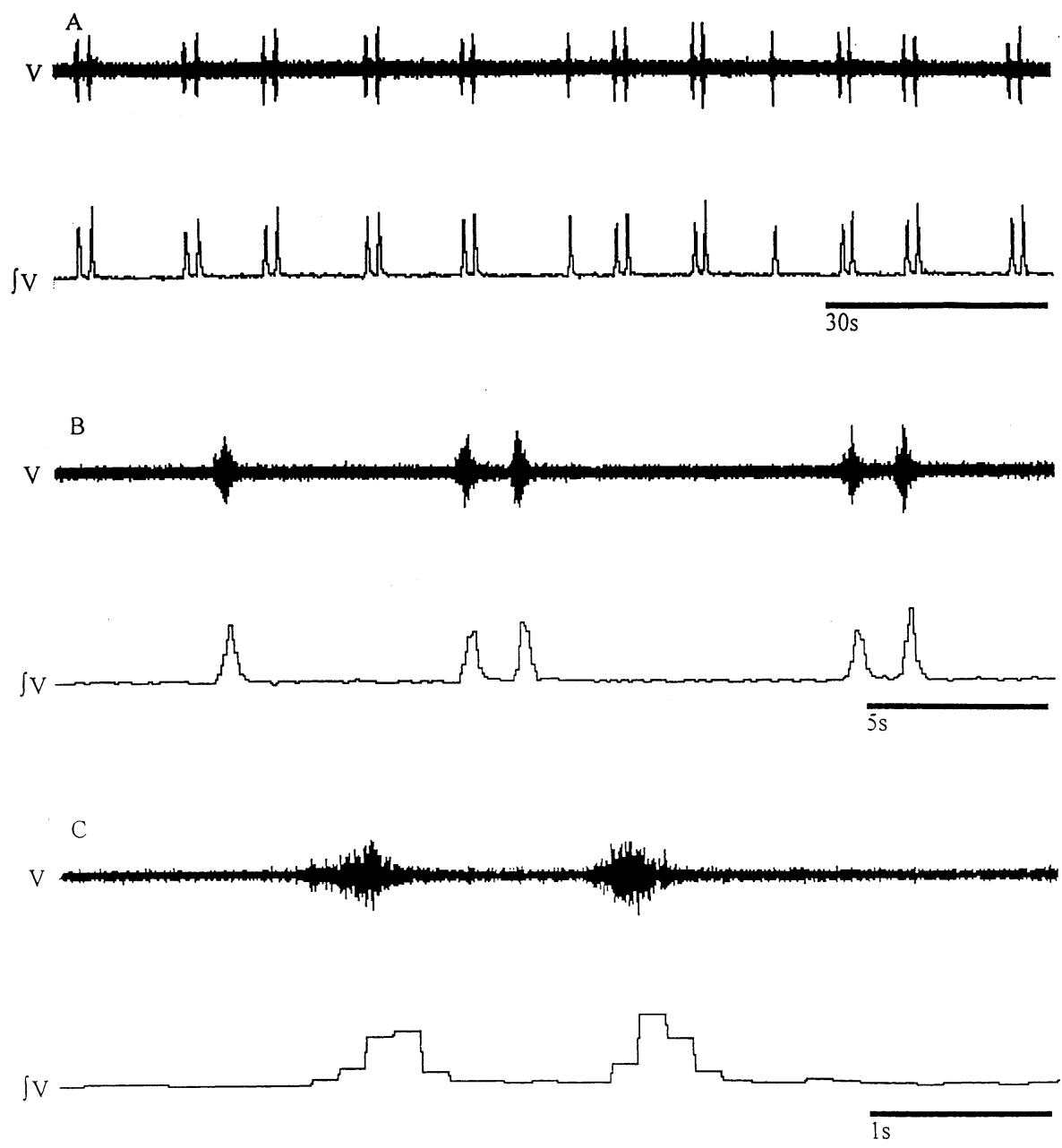

Fig. 2. Episodic motor output, in the form of doublets, recorded from the trigeminal (V) nerve root in the isolated bullfrog brainstem-spinal cord preparation. In each trace the upper figure illustrates the full wave rectified signal while the lower trace illustrates the integrated $\left(\int \mathrm{V}\right)$ signal. (A) Ten individual episodes of fictive breaths from the trigeminal nerve with two bursts per episode. Note the occurrence of several single fictive breaths. (B) An expanded trace illustrating two individual doublets and a single fictive breath. (C) A further expanded trace illustrating two fictive breaths in one doublet.

was considered to be $<1 \mathrm{sec}$ in duration. Additionally, in order for a burst to be considered a fictive breath, it had to exhibit an incrementing ('ramp-like') onset followed by a similar decrementing offset (see Fig. 3A from Kinkead and Milsom, 1997). Neural discharge longer than one second and/or exhibiting different forms of onset and/or offset was classified as an 'other' form of discharge. These 'other' forms of discharge were then sub-classified on the basis of the ten most common forms observed (see Table 1).

\subsection{Statistical analysis}

The data in Figs. 4 and 8 and Table 2 are presented as mean values \pm 1 S.E. of the mean (S.E.M). The data presented in Fig. 4 were statistically analyzed by parametric analysis of variance (ANOVA) followed by Dunnet's multiple comparison test with a single control point $(\mathrm{pH} 7.8)$. When parametric test assumptions were violated, the data were analyzed using a Kruskal-Wallis ANOVA on ranks followed by Dunnet's multiple 

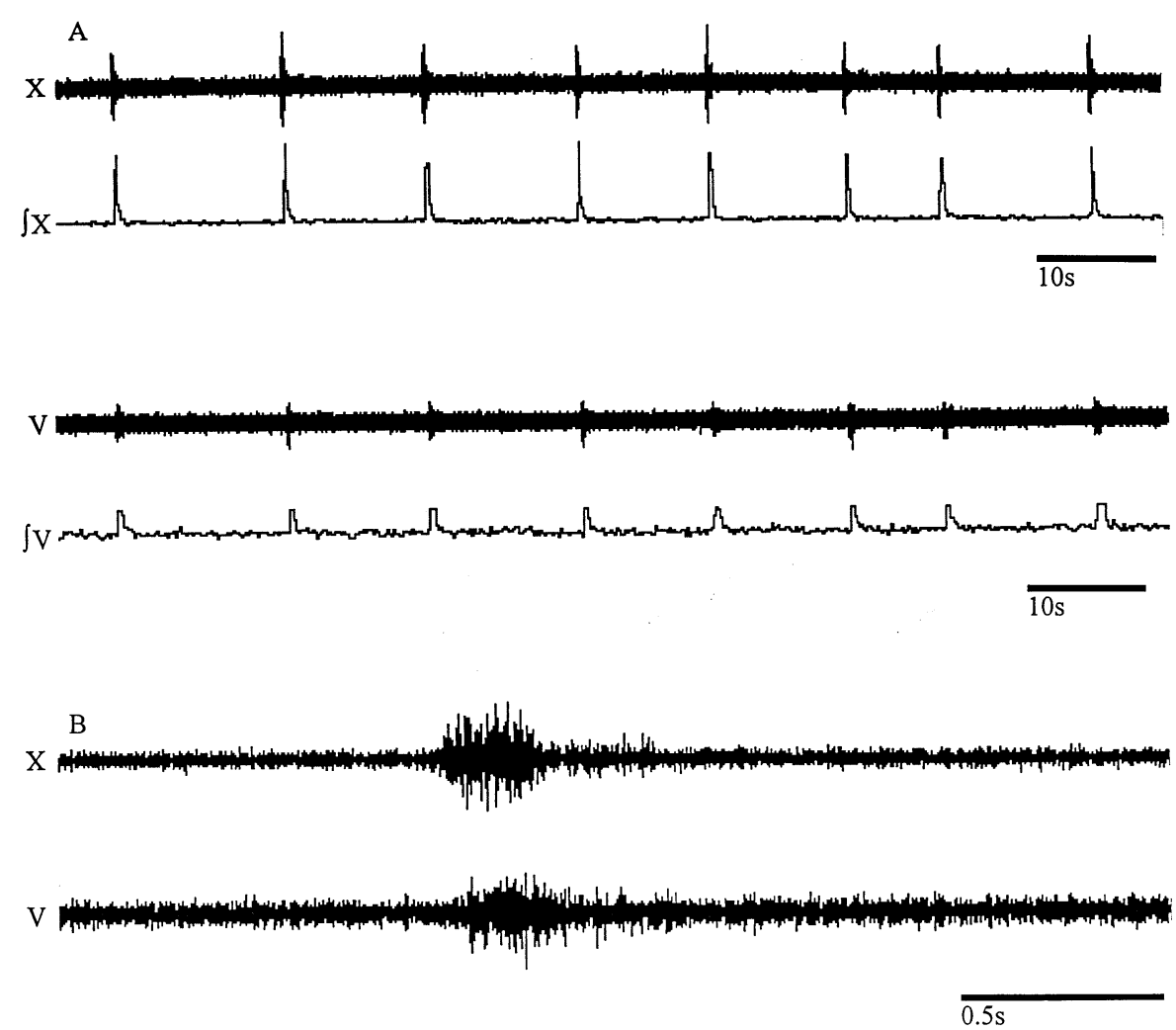

Fig. 3. (A) Non-episodic motor output recorded simultaneously from both the vagus (X) and the trigeminal (V) nerve roots. In each case, the upper trace represents the full wave rectified signal and the lower trace the integrated signal. (B) An expanded trace illustrating a single fictive breath recorded simultaneously from both the vagus and trigeminal nerves.

comparison test with a single control point $(\mathrm{pH}$ 7.8). The data presented in Fig. 8 and Table 2 were analyzed by a two sample $t$-test. All statistical testing, including determinations of normality and variance, was performed using commercial software (Sigmastat; Jandel Scientific). The fiducial limit of significance was set at $5 \%$.

\section{Results}

\subsection{Preparations with episodic and non-episodic discharge}

Nine of the 26 preparations utilized in this study exhibited motor output that was episodic (i.e. fictive breaths occurring together in groups larger than one breath; see Section 2) and, as such, was similar to that previously described by Kinkead et al. (1994). Fig. 1A illustrates in vitro motor output from the vagus nerve root with the discharge (fictive breaths) clustered into discrete episodes of 8-11 fictive breaths. Fig. 1B illustrates a single episode on an expanded scale while the incrementing and decrementing shape of the neural bursts, associated with each fictive breath, is evident from the further expanded scale in Fig. 1C. Fig. 2 illustrates another form of episodic breathing (doublets) exhibited by the isolated brainstem-spinal cord preparation. The incrementing and decrementing shape of these bursts (Fig. 2B,C) is similar to that observed in the episodes depicted in Fig. 1.

Seventeen of the 26 preparations in this study illustrated a fictive breathing pattern that was not episodic but rather consisted of relatively evenly 

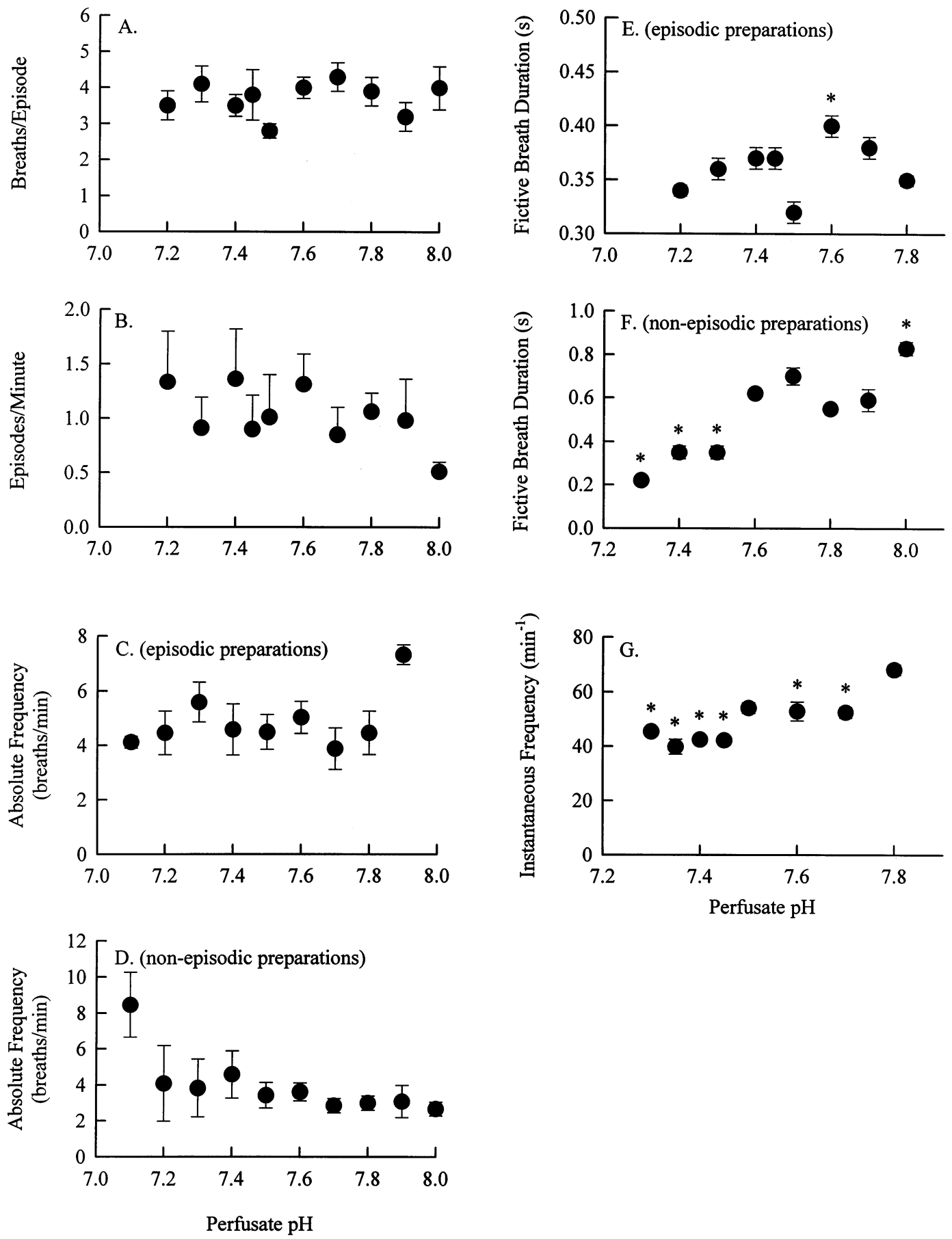

Fig. 4. The effect of altering perfusion saline $\mathrm{pH}$ on (A) the number of fictive breaths per episode, (B) the number of episodes per minute, (C and D) the absolute frequency of fictive breaths in preparations exhibiting episodic (C), and non-episodic (D) fictive breathing, $(\mathrm{E}$ and $\mathrm{F}$ ) the duration of fictive lung breaths in preparations exhibiting episodic (E), and non-episodic (F) fictive breathing and $(\mathrm{G})$ the instantaneous frequency of breaths within episodes in those preparations exhibiting episodic fictive breathing. The data are presented as the mean \pm 1 S.E. of the mean (S.E.M). An asterisk $(*)$ denotes a significant difference from the value at $\mathrm{pH} 7.8$. 
Table 1

Classification of forms of discharge from the isolated bullfrog brainstem-spinal cord preparation not associated with fictive breaths ${ }^{\text {a }}$

Shape of burst Designation Description

Type A Ramp on/off

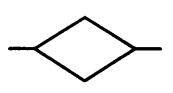

Type AA Biphasic, ramp on/off

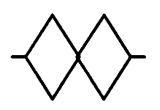

Type AAA

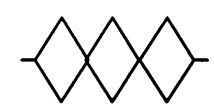

Type B

Abrupt onset

6.0

31.0

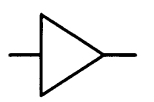

Type C

Abrupt on/off

Type D

Ramp on/off with plateau

14.0

0.0

Type BA Abrupt on, ramp on/off

6.0 7.5<smiles>CC1(C)CC12CCCC2</smiles>

Type BAA

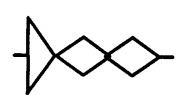

Percentage of 'abnormal bursts'

Episodic preparations Non-episodic preparations

18.0

29.0

3.0

0.0

20.0

6.0
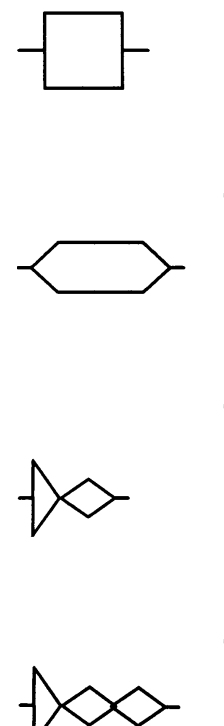
Table 1 (continued)

\begin{tabular}{clll}
\hline Shape of burst & Designation & Description & \multicolumn{2}{l}{ Percentage of 'abnormal burs's' } \\
\cline { 4 - 4 } & & Episodic preparations & Non \\
\hline RD1 & Rapid discharge & 11.0 & 6.0 \\
RD2 & Type B and rapid discharge & 0.0 & 8.0 \\
& & &
\end{tabular}

Other

11 various forms

9.0

8.0

\begin{abstract}
${ }^{a}$ Classifications are based on motor output from the vagus (X) and trigeminal (V) nerve roots. Type A bursts exhibit both a 'ramp-like' incrementing and decrementing phase similar to fictive breaths but are longer in duration. Type AA and type AAA bursts are characterised by biphasic or triphasic type A bursts, respectively. Type B bursts display an abrupt onset followed by a normal decrementing phase. Type $\mathrm{C}$ bursts display both abrupt onset and offset with a plateau phase in-between. Type $\mathrm{D}$ discharge exhibits both a ramp-like onset and offset with a plateau phase in the middle. Type BA bursts are formed by the occurrence of a type B burst followed immediately, with no pause, by a type A burst whilst a type BAA burst exhibits a biphasic type A burst following the type B burst. RD1 (rapid discharge type 1) consists of a series of normally shaped bursts occurring in very rapid succession at a frequency substantially greater than seen during the production of fictive breaths in breathing episodes. RD2 (rapid discharge Type 2) is similar to RD1 with the exception that this pattern begins with the occurrence of a type B burst. The percentage of all of the bursts represented by each of these types is reported for both the episodic and the non-episodic preparations.
\end{abstract}

spaced single breaths or the occurrence of fictive breaths in a more random pattern. An example of a non-episodic pattern of fictive breathing, recorded from both the trigeminal and vagus nerve roots, is illustrated in Fig. 3. The shape of the neural bursts associated with each fictive breath (Fig. 3B) is similar to that observed in the episodically discharging preparations.

\subsection{The effect of altering perfusion saline $\mathrm{pH}$ on fictive breathing}

The effect of altering perfusion saline $\mathrm{pH}$ on the occurrence and timing of fictive breathing is illustrated in Fig. 4. Changing the perfusion saline $\mathrm{pH}$ did not alter either the number of breaths/episode (Fig. 4A) or the number of episodes/minute (Fig. 4B) in preparations exhibiting episodic motor output. Additionally, the absolute frequency of fictive breathing was not influenced by varying $\mathrm{pH}$ in either the preparations exhibiting episodic (Fig. 4C) or non-episodic (Fig. 4D) motor output.

In those preparations exhibiting episodic discharge, fictive breath duration, with the exception of the value at $\mathrm{pH}$ 7.6, was not significantly different from the value at $\mathrm{pH} 7.8$ (Fig. 4E). On the other hand, in the non-episodic preparations, fictive breath duration at $\mathrm{pH}$ 7.3-7.5 was significantly less than that at $\mathrm{pH} 7.8$ (Fig. 4F). In the preparations exhibiting episodic motor output, the instantaneous frequency of fictive breathing within each episode also decreased as $\mathrm{pH}$ was lowered (Fig. 4G).

\subsection{Other patterns of motor output}

Motor output from various cranial nerves of the in vitro brainstem-spinal cord preparation exhibited tremendous variability regardless of whether the preparation produced episodic or 

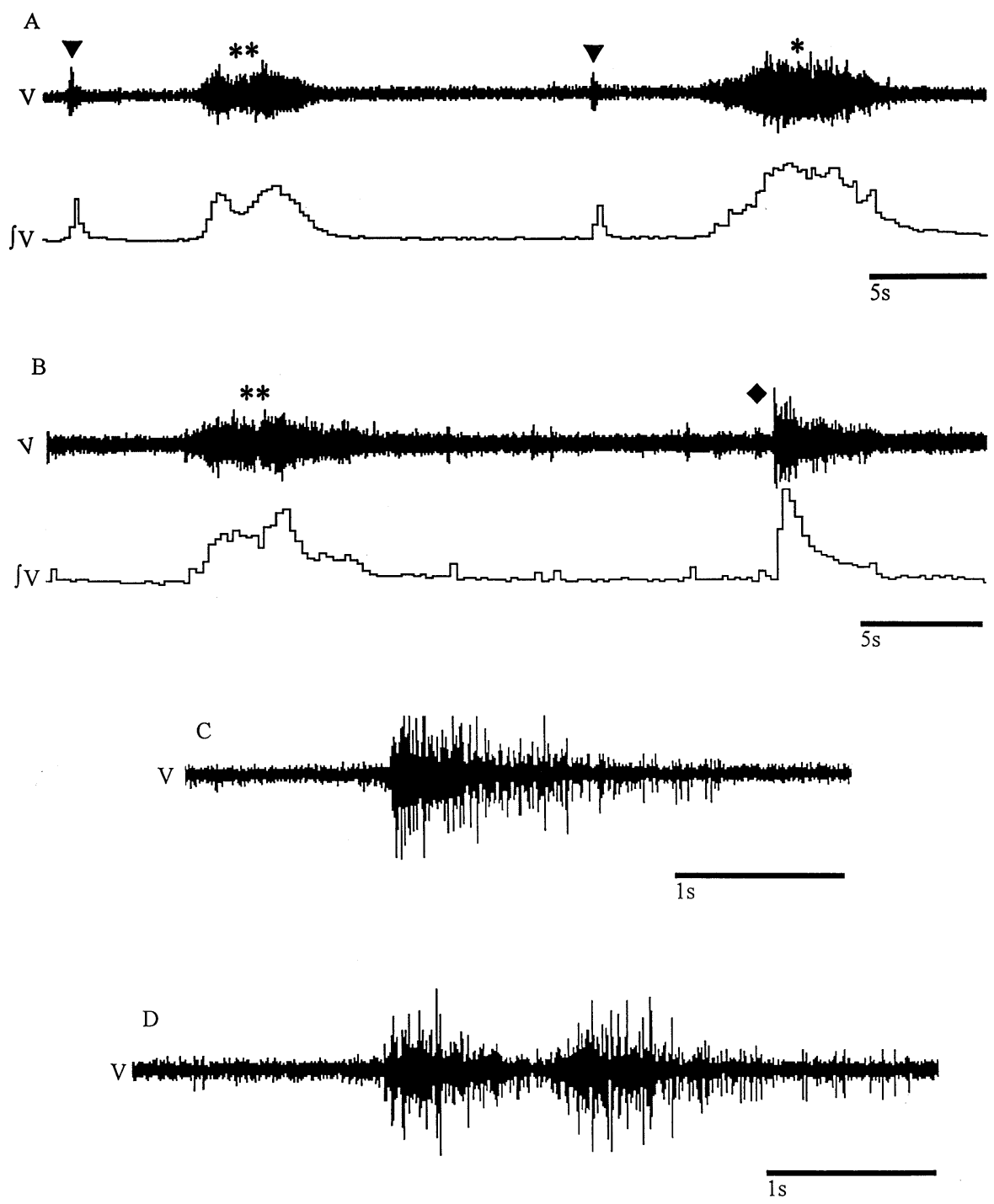

Fig. 5. (A) An illustration of two normal fictive breaths (arrowheads), a type AA (biphasic) burst (double asterisk), and a type A burst (asterisk) recorded from the trigeminal nerve root. (B) An illustration of both a type AA burst (double asterisk) and a type B burst (diamond) recorded from the trigeminal nerve root. In both A and B, the upper trace represents the full wave rectified signal while the lower trace illustrates the integrated signal. (C) An expanded trace illustrating a type B burst recorded from the trigeminal nerve. (D) An expanded trace illustrating a type AA burst recorded from the trigeminal nerve.

non-episodic patterns of fictive breathing and regardless of the perfusion saline $\mathrm{pH}$. Bursts of neural discharge with an incrementing-decrementing pattern and a duration of $<1 \mathrm{sec}$ were considered to be respiratory related (i.e. fictive breaths; Figs. 1-3) based on descriptions of motor output in decerebrate, paralyzed, unanesthetized frogs (e.g. Kogo and Remmers, 1994; Kogo et al., 1994). All other bursts of motor output were assumed to be associated with events other than normal fictive breathing.

On the basis of shape, these other forms of neural discharge have been classified into ten different categories (Table 1; Figs. 5-7). Long duration 


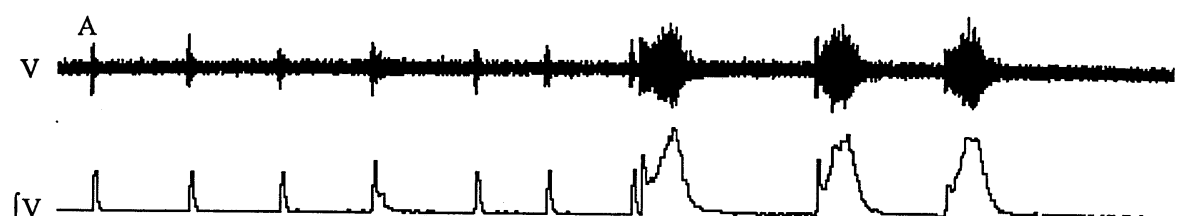

$20 \mathrm{~s}$

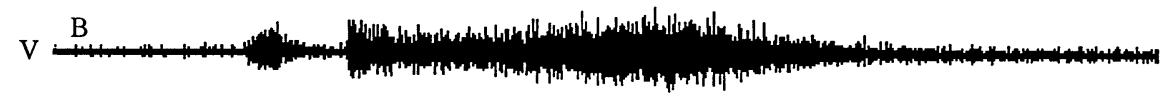

$2 \mathrm{~s}$
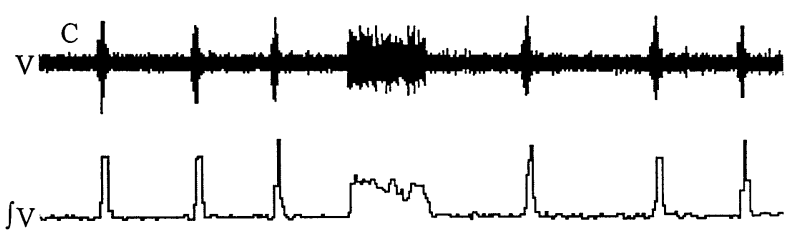

$10 \mathrm{~s}$
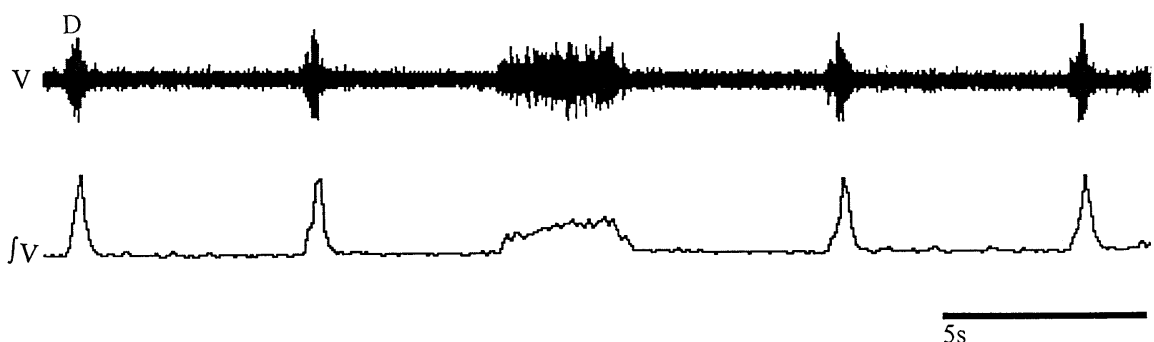

Fig. 6. (A) Seven normal fictive breaths followed by three type BA bursts recorded from the trigeminal nerve root. The upper trace illustrates the full wave rectified signal while the lower trace is the integrated waveform. (B) An expanded trace illustrating a normal fictive lung burst followed by a type BA burst from part A. Note the abrupt onset of the type BA burst which then decrements and forms a type A burst in the second half of the discharge. (C) Six normal fictive breaths and a single type C burst recorded from the trigeminal nerve root. Note the abrupt onset followed by a plateau phase and an abrupt offset. The upper trace represents the full wave rectified signal, the lower trace the integrated signal. (D) An example of a type D burst with four normal fictive breaths. Note the incrementing and decrementing phases of the type D burst with a plateau phase in between.

bursts that exhibited a ramp-like incrementing phase with a following ramp-like decrementing phase were classified as type A bursts (Table 1; Fig. 5A, single asterisk). These bursts were similar in shape to the normal fictive breaths, however they were longer in duration and often larger in magnitude. The duration of these various forms of motor output is reported in Table 2. Given the potential for variation in the signal to noise ratio between preparations, the magnitude of these neural bursts was not quantified. There was however, substantial variability in the magnitude of such discharge both between preparations and within any individual preparation. Type AA (Table 1; 

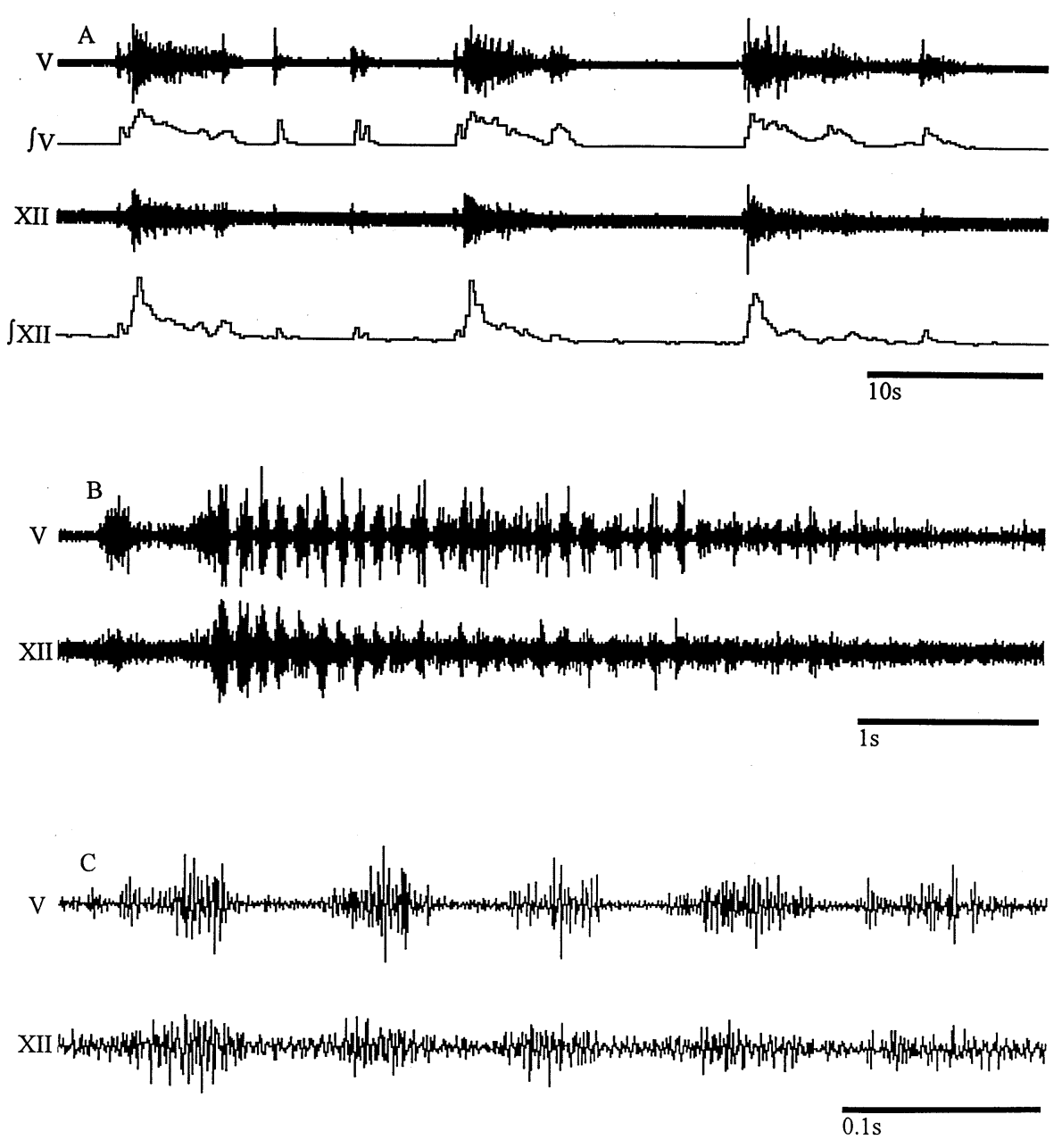

Fig. 7. An example of rapid discharge type 1 (RD1). (A) Simultaneous recordings from the trigeminal (V) and hypoglossal (XII) nerve roots. The upper traces represent the full wave rectified signal while the lower traces are the integrated nerve signals. Panels $\mathrm{B}$ and $\mathrm{C}$ represent expanded traces of the middle burst from part A. Note the occurrence of high frequency oscillations within the larger burst. Panel $\mathrm{C}$ illustrates the rapid time course of the individual bursts $(<0.1 \mathrm{sec}$ in duration) compared with the normal fictive breath duration $(<1 \mathrm{sec})$.

Fig. 5A, B, D, double asterisk) and type AAA (Table 1) bursts represent bi- or tri-phasic type A bursts, respectively.

Type B bursts (Table 1; Fig. 5B, C) were characterized by a very abrupt onset with no slow incrementing phase. A decrementing phase similar to that seen in the type A bursts, as well as in fictive breaths, then followed this abrupt onset. Type BA bursts (Table 1; Fig. 6A, B) were characterized by a type $\mathrm{B}$ burst that blended into a type A burst with no pause between the two phases. Type BAA bursts were similar to the type BA bursts but with a biphasic type A burst following the type $\mathrm{B}$ burst. Type $\mathrm{C}$ discharge (Table 1; Fig. 6C) exhibited an abrupt onset followed by a plateau level of discharge and finally an abrupt offset. Type D discharge (Table 1; Fig. 6D) exhibited an incrementing ramp-like onset, followed by a plateau phase and finally a ramp-like decrementing phase.

In some instances, large bursts of discharge that were comprised of a series of smaller bursts occur- 


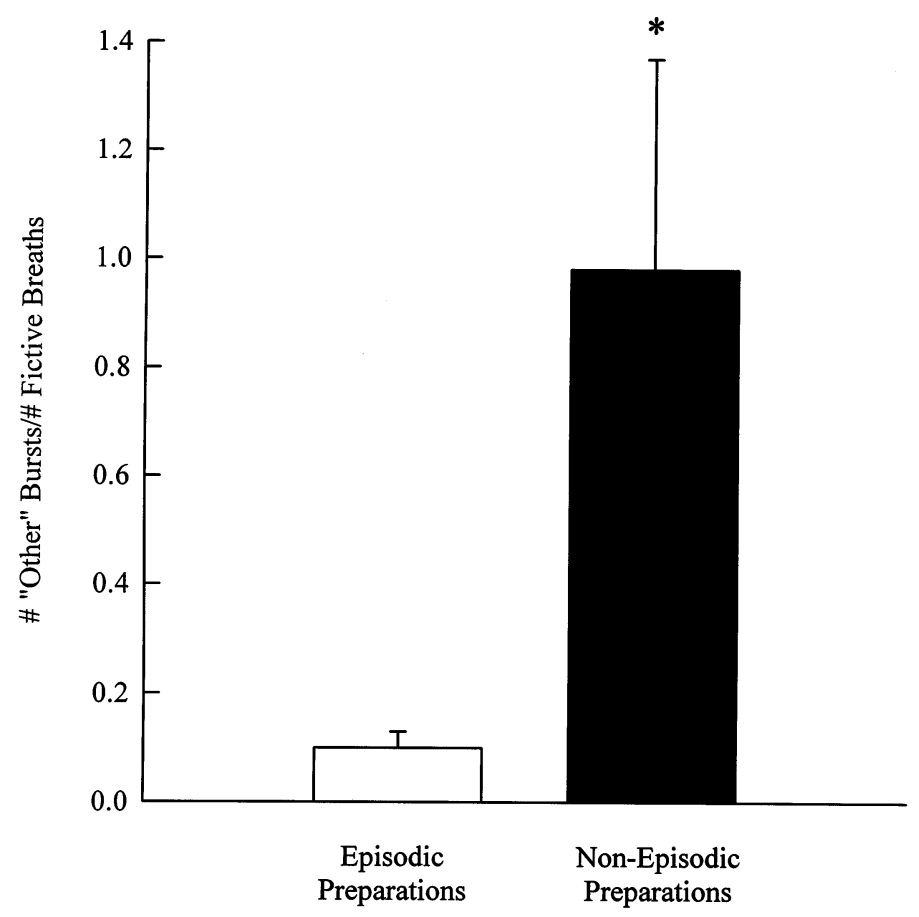

Fig. 8. The ratio of bursts of 'other patterns of neural discharge' to bursts of discharge representative of fictive breaths in the episodic (open bar) and non-episodic (solid bar) preparations. The data are presented as the mean \pm 1 S.E.M. The asterisk $(*)$ represents a statistically significant difference between the episodic and the non-episodic preparations.

ring at a very rapid frequency were observed. These burst patterns have been classified as rapid discharge one (RD1) or rapid discharge two (RD2) (see Table 1) depending upon whether they commenced with a type A or a type B burst. Fig. 7A illustrates three such large bursts which, when viewed on an expanded scale (Fig. 7B, C), are observed to consist of numerous smaller bursts which exhibit an incrementing/decrementing shape similar to that of the fictive breaths and type A bursts. The duration of these small bursts was $<0.1 \mathrm{sec}$ and the frequency of their occurrence $(\approx 10 / \mathrm{sec})$ was much greater than the frequency of fictive breaths within episodes of fictive breathing such as those illustrated in Fig. 1.

While most types of 'other' (i.e. not fictive breaths) neural activity described here were found in preparations exhibiting both episodic and nonepisodic forms of fictive breathing, their relative occurrence differed between preparations and such activity, in general, was more prevalent in the preparations exhibiting non-episodic fictive breathing (Fig. 8). Additionally, the percentage of the 'other' bursts represented by each type differed between the episodic and non-episodic preparations (Table 1) as did the duration of the type A, B, and D bursts (Table 2).

\section{Discussion}

\subsection{Preparations producing episodic and non-episodic patterns of fictive breathing}

The in vitro brainstem-spinal cord preparation is devoid of all sensory input other than that which may arise from sources contained within the brainstem-spinal cord itself. West et al. (1987) demonstrated that expression of respiratory motor output in the adult toad is conditional, suggesting that some form of elevated respiratory drive must exist in the in vitro preparation. Am- 
phibians have been shown to possess central $\mathrm{CO}_{2}$ chemoreceptors (e.g. Smatresk and Smits, 1991; Branco et al., 1992) and, despite attempts to superfuse these preparations with hyperoxic solutions, it is likely that some regions exhibit progressive degrees of hypoxia and acidosis at increasing depth from the brain surface. While Torgerson et al. (1997b) have demonstrated that no such hypoxic regions are present in an in vitro brainstem preparation from the Rana catesbieana tadpole, the brainstem was moderately acidic throughout. Finally, these preparations lack all vagal sensory input and one might expect that they would produce a fictive breathing pattern similar to that of a vagotomized animal.

The normal pattern of breathing in the bullfrog consists primarily of randomly distributed single breaths or occasional doublets that occur at a rate of $\approx 6 / \mathrm{min}$ (Kinkead and Milsom, 1997). When respiratory drive is elevated by hypoxia or hypercapnia, breathing becomes more regular and breaths frequently begin to come in episodes. While vagotomy does alter breathing pattern, in-

\section{Table 2}

Burst duration (sec) of the 'Other' forms of discharge, described in Table 1, in the episodic and non-episodic prepartions $^{\mathrm{a}}$

\begin{tabular}{lcc}
\hline $\begin{array}{l}\text { 'Other burst' } \\
\text { designation }\end{array}$ & Burst duration & \\
\cline { 2 - 3 } & $\begin{array}{l}\text { Episodic } \\
\text { preparations }\end{array}$ & $\begin{array}{c}\text { Non-episodic } \\
\text { preparations }\end{array}$ \\
& $2.1 \pm 0.3^{+}$ & $3.8 \pm 1.4^{*+}$ \\
\hline Type A & $4.8 \pm 0.8$ & $3.7 \pm 0.8$ \\
Type AA & $5.0 \pm 1.3$ & NA \\
Type AAA & $1.8 \pm 0.4$ & $3.4 \pm 0.3^{*}$ \\
Type B & $4.4 \pm 0.7$ & $3.5 \pm 0.6$ \\
Type C & $4.7 \pm 0.9$ & $7.7 \pm 1.8^{*}$ \\
Type D & $4.2 \pm 1.3$ & $5.3 \pm 0.5$ \\
Type BA & $7.8 \pm 1.5$ & $5.4 \pm 0.6$ \\
Type BAA & $11.1 \pm 2.0$ & $7.2 \pm 1.1$ \\
RD1 & $\mathrm{NA}$ & $10.1 \pm 1.3$ \\
RD2 & $9.2 \pm 1.6$ & $4.6 \pm 0.9$ \\
Other & &
\end{tabular}

\footnotetext{
a An asterisk $(*)$ denotes a significant difference between the episodic and non-episodic preparations. A plus sign $(+)$ denotes a significant difference between the Type A burst duration and the duration of the fictive breaths in either the episodic or non-episodic preparations.
}

creasing tidal volume and altering the frequency of occurrence of breathing episodes, the episodes still consist primarily of two or three breaths when the animals breath air, and increase substantially when animals become hypercapnic.

Given this background, one of the more surprising observations is the variety of fictive breathing patterns seen with the in vitro preparation in this current study and other, previously published, studies. In the work of McLean et al. (1995a,b) published records exhibit a pattern of high frequency fictive breaths that are very evenly spaced. Such a pattern of single fictive breaths might be expected from this preparation with the regularity of the breathing reflecting the removal of behavioral influences and/or peripheral inputs. The high frequency of the fictive breaths, however, is suggestive of a high level of respiratory drive, and this is always accompanied by breathing in episodes in less reduced preparations. In the work of Kinkead et al. (1994), fictive breathing did occur in episodes in vitro. Again, this is suggestive of high levels of respiratory drive and it is not clear from where this drive arises, particularly given the blunted chemosensitivity of this preparation (Kinkead et al., 1994; McLean et al., 1995a,b; Section 4.2) compared with in situ preparations and intact animals. In the present study, patterns of fictive breathing ranging from random single breaths to regular single breaths, breathing in small episodes (doublets) and breathing in large episodes, were observed. Given that all preparations were produced in an identical fashion, all we can conclude at present is that the pattern of fictive breathing from this preparation can be highly variable.

These data highlight the difference between the concept of central rhythm generation and central pattern generation. While the former refers to the ability of specialized cells, or groups of interacting cells, to initiate the central neural activity that ultimately produces each breath, the latter refers to the complex interactions that produce the spatial and temporal patterns of motor output that adjust overall ventilation in response to various inputs. The present study confirms that the central rhythm generator in the in vitro bullfrog brainstem is conditional since it is not continuously 
expressed, although it is not clear just what its activity is conditional upon. The variety of patterns of fictive breathing seen in this study suggest that even in the absence of peripheral sensory inputs, a host of central inputs must remain. Additionally, both the total level of these central inputs and the balance amongst them (i.e. inhibitory versus excitatory; positive versus negative) must vary from preparation to preparation. To date only the nucleus isthmi, located between the roof of the midbrain and the base of the cerebellum, has been identified as an important supramedullary 'central input' to medullary breathing centers in the adult bullfrog (Kinkead et al., 1997). It is possible that input from this structure may vary from preparation to preparation although currently it is not possible to speculate on how or why such input may change or what may constitute other sources of variable input.

These data also suggest that the in vitro preparation is capable of producing the complex interactions that lead to differing patterns of respiratory motor output. As such, this preparation has the potential to be a very useful tool for the study of central pattern generation in the bullfrog. On the other hand, the variety of patterns it produces, and our inability to reliably produce, or transform, any of these patterns, suggests that this preparation may have frustrating limitations for attempts to understand the mechanisms underlying the production of these patterns.

\subsection{The effect of altering perfusion saline $p H$ on fictive breathing}

With several exceptions (see Fig. 4 and discussion below), these preparations were insensitive to changes in perfusion saline $\mathrm{pH}$. The number of episodes/min, the number of breaths/episode, and the absolute frequency of fictive breathing did not change in response to a decrease to $\mathrm{pH} 7.2$ from pH 7.8 in the perfusate. The instantaneous frequency of fictive breathing within episodes as well as the duration of fictive breaths in the nonepisodic preparations were sensitive to changes in perfusion saline $\mathrm{pH}$. This suggests that there is some degree of chemosensitivity present and func- tioning in these preparations. It is unclear why some variables associated with fictive breathing would exhibit $\mathrm{pH}$ sensitivity and others would not. This differential sensitivity suggests that there are numerous processes involved in the integration of chemosensory information and its amalgamation into the production of breathing patterns. It is possible that there are different 'centers/sites' involved in controlling the overall frequency of breathing and in determining breath duration and the occurrence of fictive breaths within episodes. Such differences however, remain a matter of speculation and further study is clearly required to reconcile these observations.

Results by previous workers using in vitro preparations from adult bullfrogs or post-metamorphic tadpoles, however, have revealed a greater degree of $\mathrm{pH}$ sensitivity than observed in the current study. Thus, McLean et al. (1995a) found that the frequency of fictive breaths increased from $\approx 0.5$ to $2.9 / \mathrm{min}$ when $\mathrm{pH}$ was reduced from 8.6 to 7.4 while Kinkead et al. (1994) and Torgerson et al. (1997a) found increases from $\approx 2$ to 8 , and 0.1 to 8 breaths $/ \mathrm{min}$ over $\mathrm{pH}$ ranges from 8.3 to 7.7 and 8.4 to 7.4 , respectively. These compare with increases from 0.1 to 23 breaths/min over the $\mathrm{pH}$ range from 8.0 to 7.45 in intact animals (Kinkead and Milsom, 1994). This relative $\mathrm{pH}$ insensitivity, of the in vitro preparations, seems quite surprising at first given that there is no reason to believe that the central $\mathrm{CO}_{2}$ chemoreceptors are not intact in in vitro preparations, and that hypercapnia is such a potent respiratory stimulus in both intact animals (Kinkead and Milsom, 1994) as well as in decerebrate, paralyzed animals (Kogo et al., 1994).

The absence of any significant increase in fictive breathing frequency in the present study, however, is consistent with reports of $\mathrm{pH} / \mathrm{CO}_{2}$ insensitivity in vagotomized animals where breathing frequency either does not change or decreases as $\mathrm{pH}$ is lowered (Kinkead and Milsom, 1996, 1997). The significance of vagal feedback for the expression of central $\mathrm{pH} / \mathrm{CO}_{2}$ chemoresponses in vitro was highlighted by Kinkead et al. (1994) who observed a modest but significant increase in breathing as $\mathrm{pH}$ was lowered. However, these authors also demonstrated that phasic stimulation 
of the cut central stump of one vagus nerve, during each fictive breath, augmented the breathing frequency response threefold, returning the $\mathrm{pH} / \mathrm{CO}_{2}$ sensitivity to the range found in intact animals.

These data suggest that in the absence of vagal feedback, the in vitro brainstem preparation of the bullfrog, just like the intact frog, can only produce a modest $\mathrm{pH} / \mathrm{CO}_{2}$ chemoreflex-induced increase in fictive breathing frequency and that often even this may be absent.

\subsection{Other patterns of motor output}

Bursts of neural discharge that did not resemble fictive breaths occurred commonly from the cranial nerves monitored in the in vitro brainstem preparation of the bullfrog (see Figs. 5-7 and Table 1). These forms of discharge did not occur from one nerve preferentially over another (although the majority of the recordings in this study were from the trigeminal and vagus nerve roots). There was tremendous variability in the pattern and duration of this discharge and it did not appear to occur with any obvious rhythm. Liao et al. (1996) and Galante et al. (1996) have also reported the presence of 'complex' forms of discharge in recordings from either the facial nerve root or the facial motor nucleus in an in vitro brainstem-spinal cord preparation from the Rana catesbeiana tadpole. These discharge patterns were similar to some of those seen in the present study suggesting that their occurrence is common in both the adult and juvenile forms of this species. These authors suggested that the complex forms of activity might correspond to lung inflation cycles. This interpretation is, however, questionable since lung inflation cycles can be observed both in vitro and in situ as a series of fictive lung bursts which are all distinct (i.e. not complex) and increase in amplitude throughout the cycle (see Fig. 1D; Milsom et al., 1997).

Given that the recordings in the present study were obtained from the entire cut central ends of cranial nerves $\mathrm{V}, \mathrm{VII}, \mathrm{X}$, and $\mathrm{XII}$, there are several possible explanations for this motor activity. This activity could represent the motor output to muscles that are not involved in respiration. It could also represent motor output to muscles that are involved in both respiratory and non-respiratory events. Clearly the muscles involved in driving respiration (buccal elevators/depressors and glottal openers/closers) also function in a plethora of other processes. These include vocalisation, coughing, sneezing, swallowing, and vomiting (e.g. Grélot and Miller, 1997; Jean et al., 1997; Sakamoto et al., 1997; Shannon et al., 1997). It is quite possible that motor output not representative of fictive breathing may represent a fictive form of one of these other physiological processes. It is also possible that these other patterns of activity are representative of disrupted respiratory activity. In such a reduced preparation, it would not be too surprising to discover that some respiratory motor output was abnormal in appearance. In addition to normal fictive breathing, the isolated brain of the lamprey was found to produce several variations of the respiratory burst. These motor programs, designated 'coughs', 'arousal breathing', and 'weak breathing' were distinguished from normal fictive breaths by their much longer burst durations, distinctive underlying synaptic inputs, and separate coordinating mechanisms (Thompson, 1985).

The observation that these forms of motor output were more prevalent in the non-episodic preparations than the episodic ones may suggest that the respiratory rhythm/pattern generating circuitry, in the non-episodic preparations, is functioning in a different manner from that in the episodic preparations. Alternately, in the nonepisodic preparations, fictive breathing may be 'subservient' or suppressed by other fictive processes to a greater degree than in the episodic preparations. The long duration motor programs seen in the isolated lamprey brain could be produced independently by the caudal medulla and appeared to inhibit the production of normal fictive breaths suggesting that the caudal medulla also contained paired central pattern generators involved in lamprey respiration (Thompson, 1985). In order to fully elucidate the physiological role, if any, of these forms of discharge, it will be necessary to correlate these patterns of discharge with breathing and other behaviors in less reduced preparations. 


\section{Acknowledgements}

This study was supported by the NSERC of Canada grants to WKM. SGR was the recipient of an NSERC post-doctoral fellowship.

\section{References}

Branco, L.G.S., Glass, M.L., Hoffman, A., 1992. Central chemoreceptor drive to breathing in unanesthetized toads, Bufo paracnemis. Respir. Physiol. 87, 195-204.

Feldman, J.L., Smith, J.C., 1989. Cellular mechanisms underlying modulation of breathing pattern in mammals. Ann. New York Acad. Sci. 563, 114-130.

Feldman, J.L., Smith, J.C., Ellenberger, H.H., et al., 1990. Neurogenesis of respiratory rhythm and pattern: emerging concepts. Am. J. Physiol. 259, 879-886.

Feldman, J.L., Smith, J.C., Liu, G., 1991. Respiratory pattern formation in mammals: in vitro en bloc analyses. Curr. Opin. Neurobiol. 1, 590-594.

Galante, R.J., Kubin, L., Fishman, A.P., Pack, A.I., 1996. Role of chloride-mediated inhibition in respiratory rhythmogenesis in an in vitro brainstem of tadpole, Rana catesbeiana. J. Physiol. Lond. 492, 545-558.

Grélot, L., Miller, A.D., 1997. Neural control of respiratory muscle activation during vomiting. In: Miller, A.D., Bianchi, A.L., Bishop, B.P. (Eds.), Neural control of the respiratory muscles. CRC Press, Boca Raton FL, pp. 239-248.

Jean, A., Car, A., Kessler, J.P., 1997. Brainstem organisation of swallowing and its interaction with respiration. In: Miller, A.D., Bianchi, A.L., Bishop, B.P. (Eds.), Neural control of the respiratory muscles. CRC Press, Boca Raton FL, pp. 223-238.

Kinkead, R., Filmyer, W.G., Mitchell, G.S., Milsom, W.K., 1994. Vagal input enhances responsiveness of respiratory discharge to central changes in $\mathrm{pH} / \mathrm{CO}_{2}$ in bullfrogs. J. Appl. Physiol. 77, 2048-2051.

Kinkead, R., Milsom, W.K., 1994. Chemoreceptors and control of episodic breathing in the bullfrog (Rana catesbeiana). Respir. Physiol. 95, 81-98.

Kinkead, R., Milsom, W.K., 1996. $\mathrm{CO}_{2}$-sensitive olfactory and pulmonary receptor modulation of episodic breathing in bullfrogs. Am. J. Physiol. 270, R134-R144.

Kinkead, R., Harris, M.B., Milsom, W.K., 1997. The role of the nucleus isthmi in respiratory pattern formation in bullfrogs. J. Exp. Biol. 200, 1781-1793.

Kinkead, R., Milsom, W.K., 1997. Role of pulmonary stretch receptor feedback in control of episodic breathing in the bullfrog. Am. J. Physiol. 272, R497-R508.

Kogo, N., Perry, S.F., Remmers, J.E., 1994. Neural organization of the ventilatory activity of the frog, Rana catesbeiana I. J. Neurobiol. 25, 1067-1079.
Kogo, N., Remmers, J.E., 1994. Neural organization of the ventilatory activity in the frog, Rana catesbeiana II. J. Neurobiol. 25, 1080-1094.

Liao, G.-S., Kubin, L., Galante, R.J., Fishman, A.P., Pack, A.I., 1996. Respiratory activity in the facial nucleus in an in vitro brainstem of the tadpole, Rana catesbeiana. J. Physiol. Lond. 492, 529-544.

McLean, H.A., Kimura, N., Kogo, N., Perry, S.F., Remmers, J.E., 1995a. Fictive respiratory rhythm in the isolated brainstem of bullfrogs. J. Comp. Physiol. A. 176, 703-713.

McLean, H.A., Perry, S.F., Remmers, J.E., 1995b. Two regions in the isolated brainstem of the frog that modulate respiratory-related activity. J. Comp. Physiol. A. 177, 145158.

Milsom, W.K., Harris, M.B., Reid, S.G., 1997. Do descending influences alternate to produce episodic breathing? Respir. Physiol. 110, 307-317.

Perry, S.F., McLean, H.A., Kogo, N., et al., 1995. The frog brainstem preparation as a model for studying the central control of breathing in tetrapods. Braz. J. Med. Biol. Res. 28, $1-8$.

Reid, S.G., Milsom, W.K., 1996. Respiratory pattern generation by the isolated bullfrog brainstem-spinal cord, Physiologist 39, A5.8.

Sakakibara, Y., 1984a. The pattern of respiratory nerve activity in the bullfrog. Jpn. J. Physiol. 34, 269-282.

Sakakibara, Y., 1984b. Trigeminal nerve activity and buccal pressure as an index of total inspiratory activity in the bullfrog. Jpn. J. Physiol. 34, 827-838.

Sakamoto, T., Nonaka, S., Katada, A., 1997. Control of respiratory muscles during speech and vocalisation. In: Miller, A.D., Bianchi, A.L., Bishop, B.P. (Eds.), Neural control of the respiratory muscles. CRC Press, Boca Raton FL, pp. 249-258.

Shannon, R., Bolser, D.C., Lindsey, B.G., 1997. Neural control of sneezing and coughing. In: Miller, A.D., Bianchi, A.L., Bishop, B.P. (Eds.), Neural control of the respiratory muscles. CRC Press, Boca Raton FL, pp. 213-222.

Smatresk, N., Smits, A.W., 1991. Effects of central and peripheral chemoreceptor stimulation on ventilation in the marine toad, Bufo marinus. Respir. Physiol. 83, 223-238.

Suzue, T., 1984. Respiratory rhythm generation in the in vitro brainstem-spinal cord preparation of the neonatal rat. J. Physiol. Lond. 384, 173-183.

Thompson, K.J., 1985. Organization of inputs to motoneurons during fictive respiration in the isolated lamprey brain. J. Comp. Physiol. A. 157, 291-302.

Torgerson, C.S., Gdovin, M.J., Remmers, J.E., 1997a. Ontogeny of central chemoreception during fictive gill and lung ventilation in an in vitro brainstem preparation of Rana catesbeiana. J. Exp. Biol. 200, 2063-2072.

Torgerson, C.S., Gdovin, M.J., Kogo, N., Remmers, J.E., 1997b. Depth profiles of $\mathrm{pH}$ and $\mathrm{PO}_{2}$ in the in vitro brainstem preparation of the tadpole, Rana catesbeiana. Respir. Physiol. 108, 205-213.

West, N.H., Topor, Z.L., van Vliet, B.N., 1987. Hypoxemic threshold for lung ventilation in the toad. Respir. Physiol. 70, 377-390. 